\title{
Melanoma-Targeting Property of Y-90-Labeled Lactam-Cyclized $\alpha$-Melanocyte-Stimulating Hormone Peptide
}

\author{
Jingli Xu, ${ }^{1}$ Jianquan Yang, ${ }^{1}$ Rene Gonzalez, ${ }^{2}$ Darrell R. Fisher, ${ }^{3}$ and Yubin Miao ${ }^{1}$
}

\begin{abstract}
Purpose: The purpose of this study was to evaluate melanoma-targeting property of ${ }^{90} \mathrm{Y}$-DOTA-GGNle$\mathrm{CycMSH}_{\text {hex }}$ to facilitate its potential therapeutic application.

Materials and Methods: DOTA-GGNle-CycMSH ${ }_{\text {hex }}$ was synthesized and readily labeled with ${ }^{90} \mathrm{Y}$ in $0.25 \mathrm{M}$ $\mathrm{NH}_{4}$ Ac-buffered solution to generate ${ }^{90} \mathrm{Y}$-DOTA-GGNle-CycMSH $\mathrm{Hex}_{\text {he }}$ The specific receptor binding, internalization, and efflux of ${ }^{90} \mathrm{Y}-\mathrm{DOTA}-\mathrm{GGNle}-\mathrm{CycMSH}_{\text {hex }}$ were determined on B16/F10 murine melanoma cells. The biodistribution property of ${ }^{90} \mathrm{Y}-\mathrm{DOTA}-\mathrm{GGNle}-\mathrm{CycMSH}_{\text {hex }}$ was examined on B16/F10 melanoma-bearing C57 mice.

Results: ${ }^{90}$ Y-DOTA-GGNle-CycMSH ${ }_{\text {hex }}$ displayed receptor-specific binding, rapid internalization, and prolonged efflux on B16/F10 melanoma cells. ${ }^{90} \mathrm{Y}-\mathrm{DOTA}-\mathrm{GGNle-CycMSH} \mathrm{Hex}_{\text {ex }}$ exhibited high uptake and prolonged retention in melanoma, and fast urinary clearance on B16/F10 melanoma-bearing C57 mice. The B16/ F10 tumor uptake was $20.73 \% \pm 7.99 \%, 19.93 \% \pm 5.73 \%, 14.8 \% \pm 4.61 \%$, and $6.69 \% \pm 1.85 \% \mathrm{ID} / \mathrm{g}$ at $0.5,2,4$, and $24 \mathrm{~h}$ postinjection, respectively.

Conclusions: ${ }^{90} \mathrm{Y}-\mathrm{DOTA}-\mathrm{GGNle}-\mathrm{CycMSH}_{\mathrm{hex}}$ displayed melanocortin-1 receptor (MC1R) targeting and specificity on B16/F10 melanoma cells and tumors. The favorable melanoma-targeting property and fast urinary clearance of ${ }^{90}$ Y-DOTA-GGNle-CycMSH $\mathrm{M}_{\text {hex }}$ warranted its evaluation for melanoma therapy in future studies.
\end{abstract}

Keywords: melanocortin-1 receptor, melanoma therapy, ${ }^{90} \mathrm{Y}-\mathrm{DOTA}-\mathrm{GGNle}-\mathrm{CycMSH}_{\mathrm{hex}}$

\section{Introduction}

$\mathbf{M}$ alignant melanoma is the most lethal skin cancer and fifth most commonly diagnosed cancer in the United States with $\sim 96,480$ new cases in $2019 .{ }^{1}$ Extreme aggressiveness of metastatic melanoma leads to high mortality among metastatic melanoma patients. Despite the promising result that the median overall survival of metastatic melanoma patients has been improved by months through new treatments such as Vemurafenib (BRAF inhibitor), ipilimumab (targeting CTLA-4), and Nivolumab (PD-1 inhibitor), ${ }^{2-6}$ the treatments are far from satisfactory due to the low long-term survival $(<10 \%)$ for metastatic melanoma patients. There is an urgent need to develop new therapeutic agents for melanoma.

Melanocortin-1 receptor (MC1R) is an attractive G protein-coupled receptor (GPCR), which overexpresses on both murine and human melanoma cells. ${ }^{7-12}$ Importantly, $>80 \%$ of amelanotic and melanotic human metastatic melanoma samples exhibit MC1Rs. ${ }^{7}$ Recently, we have demonstrated the clinical relevance of MC1R for melanoma imaging through the first-in-human study of ${ }^{68} \mathrm{Ga}$-DOTA-GGNle-CycMSH ${ }_{\text {hex }}$ (1,4,7,10-tetraazacyclononane-1,4,7,10-tetraacetic acid-GlyGly-Nle-c[Asp-His-DPhe-Arg-Trp-Lys]-CONH ${ }_{2}$, which targets MC1Rs. ${ }^{13}$ Remarkably, ${ }^{68}$ Ga-DOTA-GGNle-CycMSH ${ }_{\text {hex }}$ positron emission tomography (PET) could clearly visualize the melanoma metastases in brain, lung, connective tissue, and small intestine of melanoma patients. ${ }^{13}$ These exciting first-in-human images of melanoma metastases highlighted the potential of MC1R as a melanoma target for targeted radionuclide therapy.

We have been interested in utilizing therapeutic radionuclides to target MC1Rs for melanoma therapy. In our previous report, we determined the melanoma-targeting property

Departments of ${ }^{1}$ Radiology and ${ }^{2}$ Medical Oncology, University of Colorado Denver, Aurora, Colorado.

${ }^{3}$ Versant Medical Physics and Radiation Safety, Richland, Washington.

Address correspondence to: Yubin Miao; Department of Radiology, School of Medicine, University of Colorado Denver; 12700 East 19th Avenue, MS C278, Aurora, CO 80045

E-mail: yubin.miao@cuanschutz.edu 
of ${ }^{177}$ Lu-DOTA-GGNle-CycMSH ${ }_{\text {hex }}$ on B16/F1 melanomabearing C57 mice. $^{14}$ Interestingly, ${ }^{177}$ Lu-DOTA-GGNle$\mathrm{CycMSH}_{\text {hex }}$ displayed high B16/F1 melanoma uptake of $20.25 \% \pm 4.59 \%$ and $21.63 \% \pm 6.27 \% \mathrm{ID} / \mathrm{g}$ at 0.5 and $2 \mathrm{~h}$ postinjection, respectively. Meanwhile, the melanoma lesions were clearly visualized using ${ }^{177}$ Lu-DOTA-GGNle$\mathrm{CycMSH}_{\text {hex }}$ as an imaging probe. ${ }^{14}$ In this study, we are interested in replacing ${ }^{177} \mathrm{Lu}$ with ${ }^{90} \mathrm{Y}$ to examine whether the change of therapeutic radionuclide could still maintain favorable melanoma-targeting property of ${ }^{90} \mathrm{Y}$-DOTA-GGNle$\mathrm{CycMSH}_{\text {hex }}{ }^{90} \mathrm{Y}$ is a high-energy $\beta$-particle emitter with a maximum $\beta$ energy of $2.3 \mathrm{MeV}$ and a half-life of 2.7 days. Specifically, we prepared ${ }^{90}$ Y-DOTA-GGNle-CycMSH ${ }_{\text {hex }}$ and determined its MC1R-targeting property on B16/F10 melanoma cells and tumor-bearing mice.

\section{Materials and Methods}

\section{Chemicals and reagents}

Amino acids, DOTA-tri-tert-butyl ester, and resin were purchased from Advanced ChemTech, Inc. (Louisville, KY), Macrocyclics, Inc. (Richardson, TX) and Novabiochem (San Diego, CA) for peptide synthesis, respectively. ${ }^{90} \mathrm{YCl}_{3}$ was purchased from PerkinElmer Health Sciences, Inc. (Waltham, MA) for radiolabeling and biodistribution. MC1R antibody (Rabbit/IgG) and FITC-conjugated antirabbit secondary antibody were purchased from Thermo Scientific (Rockford, IL) for MC1R staining on melanoma cells and tumors. All other chemicals used in this study were purchased from Thermo Fisher Scientific (Waltham, MA) and used without further purification. Four percent paraformaldehyde (PFA) in phosphate-buffered saline (PBS) was obtained from Alfa Aesar (Ward Hill, MA), xylene was obtained from Fisher Chemical (Fair Lawn, NJ), 4',6-diamidino-2phenylindole (DAPI) Fluoromount-G mounting medium was obtained from SouthernBiotech (Birmingham, AL), and Prolong Diamond antifade mounting reagent with DAPI was obtained from Life Technologies (Eugene, OR). B16/F10 murine melanoma cells were obtained from American Type Culture Collection (Manassas, VA).

\section{Preparation, serum stability, and specific binding} of ${ }^{90} \mathrm{Y}$-DOTA-GGNle-CycMSH $\mathrm{H}_{\text {hex }}$

DOTA-GGNle-CycMSH $\mathrm{CH}_{\text {hex }}$ was synthesized using standard fluorenylmethyloxycarbonyl (Fmoc) chemistry and characterized by liquid chromatography-mass spectrometry. ${ }^{15}{ }^{90} \mathrm{Y}$-DOTA-GGNle-CycMSH ${ }_{\text {hex }}$ was prepared in a $0.25 \mathrm{M} \mathrm{NH}_{4} \mathrm{OAc}$-buffered solution ( $\mathrm{pH} 4.5$ ). In brief, $30 \mu \mathrm{L}$ of ${ }^{90} \mathrm{YCl}_{3}(37-74 \mathrm{MBq}$ in $0.05 \mathrm{M} \mathrm{HCl}$ aqueous solution), $10 \mu \mathrm{L}$ of $1 \mathrm{mg} / \mathrm{mL}$ peptide aqueous solution, and $200 \mu \mathrm{L}$ of $0.25 \mathrm{M} \mathrm{NH}_{4} \mathrm{OAc}$ were added to a reaction vial and incubated at $75^{\circ} \mathrm{C}$ for $30 \mathrm{~min}$. After the incubation, $10 \mu \mathrm{L}$ of $0.5 \%$ EDTA (ethylenediaminetetraacetic acid) aqueous solution was added to the reaction vial to scavenge potential unbound ${ }^{90} \mathrm{Y}^{3+}$ ions. The radiolabeled complexes were purified to single species by Waters RP-HPLC (Milford, MA) on a Grace Vydac C-18 reverse-phase analytical column (Deerfield, IL) using the following gradient at a $1 \mathrm{~mL} / \mathrm{min}$ flowrate. The mobile phase consisted of solvent $\mathrm{A}(20 \mathrm{mM} \mathrm{HCl}$ aqueous solution) and solvent $\mathrm{B}\left(100 \% \mathrm{CH}_{3} \mathrm{CN}\right)$. The gradient was initiated and kept at 82:18 A/B for 3 min followed by a linear gradient of $82: 18 \mathrm{~A} / \mathrm{B}$ to $72: 28 \mathrm{~A} / \mathrm{B}$ over $20 \mathrm{~min}$. Then, the gradient was changed from $72: 28 \mathrm{~A} / \mathrm{B}$ to 10:90 $\mathrm{A} / \mathrm{B}$ over $3 \mathrm{~min}$ followed by an additional $5 \mathrm{~min}$ at $10: 90 \mathrm{~A} / \mathrm{B}$. Thereafter, the gradient was changed from $10: 90 \mathrm{~A} / \mathrm{B}$ to $82: 18 \mathrm{~A} / \mathrm{B}$ over $3 \mathrm{~min}$. The purified peptide was purged with $\mathrm{N}_{2}$ gas for $15 \mathrm{~min}$ to remove the acetonitrile. The $\mathrm{pH}$ of the final solution was adjusted to 7.4 with $0.1 \mathrm{~N} \mathrm{NaOH}$ and sterile saline for animal studies.

In vitro serum stability of ${ }^{90} \mathrm{Y}$-DOTA-GGNle-CycMSH $\mathrm{CHex}_{\mathrm{h}}$ was determined by incubation in mouse serum at $37^{\circ} \mathrm{C}$ for $4 \mathrm{~h}$ and monitored for degradation by Reversed-phase highperformance liquid chromatography (RP-HPLC). The specific binding of ${ }^{90} \mathrm{Y}$-DOTA-GGNle-CycMSH $\mathrm{CH}_{\text {hex }}$ was determined on B16/F10 melanoma cells. In brief, the B16/F10 cells $\left(1 \times 10^{6}\right.$ cells/tube, $\left.n=3\right)$ were incubated at $25^{\circ} \mathrm{C}$ for $2 \mathrm{~h}$ with $\sim 0.037 \mathrm{MBq}$ of ${ }^{90} \mathrm{Y}$-DOTA-GGNle-CycMSH $\mathrm{H}_{\text {hex }}$ with or without $10 \mu \mathrm{g}(6.07 \mathrm{nmol})$ of unlabeled $\left[\mathrm{Nle}^{4}\right.$, D$\left.\mathrm{Phe}^{7}\right]-\alpha-\mathrm{MSH}$ (NDP-MSH) in $0.3 \mathrm{~mL}$ of binding medium [Modified Eagle's medium with $25 \mathrm{mM} \mathrm{N}$-(2-hydroxyethyl)piperazine- $N^{\prime}$-(2-ethanesulfonic acid), $\mathrm{pH} 7.4,0.2 \%$ bovine serum albumin (BSA), $0.3 \mathrm{mM} \mathrm{1,10-phenathroline].} \mathrm{After}$ the incubation, the cells were rinsed three times with $0.5 \mathrm{~mL}$ of ice-cold $\mathrm{pH} \mathrm{7.4,0.2 \%} \mathrm{BSA/0.01} \mathrm{M} \mathrm{PBS} \mathrm{and} \mathrm{measured} \mathrm{in}$ a Wallac 1480 automated $\gamma$ counter (PerkinElmer, NJ).

\section{Internalization and efflux of ${ }^{90} \mathrm{Y}$-DOTA-GGNle-CycMSH $\mathrm{Hex}_{\text {hex }}$}

Cellular internalization and efflux of ${ }^{90}$ Y-DOTA-GGNle$\mathrm{CycMSH}_{\text {hex }}$ were evaluated on B16/F10 melanoma cells. The B16/F10 cells $\left(3 \times 10^{5} /\right.$ well $)$ were seeded onto a 24 -well cell culture plate and incubated at $37^{\circ} \mathrm{C}$ overnight. After being washed once with binding medium, the cells were incubated at $25^{\circ} \mathrm{C}$ for $20,40,60,90$, and $120 \mathrm{~min}(n=3)$ in the presence of $\sim 130,000$ counts per minute of HPLC purified ${ }^{90}$ Y-DOTA-GGNle-CycMSH $\mathrm{H}_{\text {hex }}$. After incubation, the cells were rinsed with $2 \times 0.5 \mathrm{~mL}$ of ice-cold $\mathrm{pH} 7.4,0.2 \%$ BSA/0.01 M PBS. Cellular internalization of ${ }^{90}$ Y-DOTAGGNle-CycMSH $\mathrm{Hex}_{\text {hex }}$ was assessed by washing the cells with acidic buffer ( $40 \mathrm{mM}$ sodium acetate [pH 4.5] containing $0.9 \%$ $\mathrm{NaCl}$ and $0.2 \%$ BSA) to remove the membrane-bound radioactivity. The remaining internalized radioactivity was obtained by lyzing the cells with $0.5 \mathrm{~mL}$ of $1 \mathrm{~N} \mathrm{NaOH}$ for $5 \mathrm{~min}$. Membrane-bound and internalized ${ }^{90} \mathrm{Y}$ activity was counted in a $\gamma$ counter. Cellular efflux of ${ }^{90} \mathrm{Y}$-DOTA-GGNle-CycMSH $\mathrm{H}_{\text {hex }}$ was determined by incubating cells with ${ }^{90} \mathrm{Y}$-DOTA-GGNle$\mathrm{CycMSH}_{\text {hex }}$ at $25^{\circ} \mathrm{C}$ for $2 \mathrm{~h}$, removing nonspecific bound activity with $2 \times 0.5 \mathrm{~mL}$ of ice-cold $\mathrm{pH} 7.4,0.2 \% \mathrm{BSA} / 0.01 \mathrm{M}$ PBS rinse, and monitoring radioactivity released into cell culture medium. At time points of 20, 40, 60, 90, and $120 \mathrm{~min}$, the radioactivity on cell surface and in cells was separately collected and counted in a $\gamma$ counter.

\section{MC1R staining on B16/F10 melanoma cells and lesions}

The B16/F10 cells $\left(1 \times 10^{5}\right.$ cells/well $)$ were seeded onto a 4-well Lab-Tek Chamber Glass Slide System (Thermo Scientific, MA) and incubated at $37^{\circ}$ overnight. After $24 \mathrm{~h}$, the cells were fixed with 4\% PFA in PBS and incubated at room temperature for $15 \mathrm{~min}$, washed with PBS three times, treated with $0.5 \%$ Triton $\mathrm{X}-100$ at room temperature for $15 \mathrm{~min}$, and washed with PBS three times. The cells were 
incubated with MC1R antibody (1:300 dilution) at room temperature for $1 \mathrm{~h}$ followed by PBS wash three times, then incubated with FITC-conjugated antirabbit secondary antibody (1:100 dilution) at room temperature for $30 \mathrm{~min}$ followed by PBS wash three times. The cells were stained for nuclei and mounted with DAPI Fluoromount-G mounting medium (SouthernBiotech) and stayed in the dark at room temperature for $24 \mathrm{~h}$. The fluorescent signal was observed and recorded at $100 \times$ magnification under an Olympus FV1000 confocal microscope.

All animal studies were conducted in compliance with Institutional Animal Care and Use Committee approval. B16/F10 flank melanoma-bearing mice were generated for MC1R staining and biodistribution studies. In brief, each C57 mouse was subcutaneously inoculated with $1 \times 10^{6}$ B16/F10 cells on the right flank. Ten days postinoculation, the tumor weights reached $\sim 0.2 \mathrm{~g}$. The B16/F10 tumor was used to generate paraffin-embedded tumor sections ( $5 \mu \mathrm{m}$ thickness) for MC1R staining. The paraffin-embedded tumor sections were deparaffinized with xylene first, and incubated with MC1R antibody (1:300 dilution) at room temperature for $1 \mathrm{~h}$ followed by PBS wash three times, then incubated with FITC-conjugated antirabbit secondary antibody (1:100 dilution) at room temperature for $30 \mathrm{~min}$ followed by PBS wash three times. Tissue samples were stained and mounted with Prolong Diamond antifade mounting reagent with DAPI (Life Technologies). The fluorescent signal was observed and recorded at $100 \times$ magnification under an Olympus FV1000 confocal microscope.

\section{Biodistribution and bremsstrahlung imaging} of ${ }^{90} \mathrm{Y}$-DOTA-GGNle-CycMSH $\mathrm{Hex}_{\mathrm{H}}$

The biodistribution property of ${ }^{90} \mathrm{Y}$-DOTA-GGNle$\mathrm{CycMSH}_{\text {hex }}$ was determined on B16/F10 flank melanomabearing C57 mice (Charles River, Wilmington, MA). Each melanoma-bearing mouse was injected with $0.037 \mathrm{MBq}$ of ${ }^{90}$ Y-DOTA-GGNle-CycMSH ${ }_{\text {hex }}$ through the tail vein. Mice were sacrificed at $0.5,2,4$, and $24 \mathrm{~h}$ postinjection, and tumors and organs of interest were harvested, weighed, and counted. Blood values were taken as $6.5 \%$ of the whole-body weight. The specificity of the tumor uptake of ${ }^{90}$ Y-DOTA-GGNle$\mathrm{CycMSH}_{\text {hex }}$ was determined by coinjecting $10 \mu \mathrm{g}(6.07 \mathrm{nmol})$ of unlabeled NDP-MSH, which is a linear $\alpha-\mathrm{MSH}$ peptide analog with subnanomolar MC1R binding affinity.

We were interested whether B16/F10 melanoma lesions could be imaged by single photon emission computed tomography (SPECT) by collecting ${ }^{90} \mathrm{Y}$ bremsstrahlung photons. As an exploratory effort, we examined the bremsstrahlung imaging property of ${ }^{90}$ Y-DOTA-GGNle$\mathrm{CycMSH}_{\text {hex }}$ on B16/F10 flank melanoma-bearing C57 mice using a small energy window of $126.5-155.7 \mathrm{keV}$. Each melanoma-bearing mouse was injected with $7.4 \mathrm{MBq}$ of ${ }^{90}$ Y-DOTA-GGNle-CycMSH$_{\text {hex }}$ through the tail vein. SPECT imaging study was performed at $2 \mathrm{~h}$ postinjection. CT data were collected followed by SPECT data acquisition. Reconstructed SPECT/CT data were visualized using Vivoquant (Invicro, Boston, MA).

\section{Results}

${ }^{90}$ Y-DOTA-GGNle-CycMSH ${ }_{\text {hex }}$ (Fig. 1) was readily prepared with $>95 \%$ radiolabeling yield, and was completely separated from its excess nonlabeled peptide by RP-
HPLC. The retention time of ${ }^{90}$ Y-DOTA-GGNle$\mathrm{CycMSH}_{\text {hex }}$ and DOTA-GGNle-CycMSH $\mathrm{CH}_{\text {hex }}$ was 19.3 and $17.1 \mathrm{~min}$, respectively. The specific activity of ${ }^{90} \mathrm{Y}$-DOTA$\mathrm{GGNle}-\mathrm{CycMSH}_{\text {hex }}$ was $\sim 4.8962 \times 10^{4} \mathrm{mCi} / \mu \mathrm{mol} .{ }^{90} \mathrm{Y}-$ DOTA-GGNle-CycMSH hex was stable in mouse serum at $37^{\circ} \mathrm{C}$ for $4 \mathrm{~h}$ (Fig. 2). ${ }^{90}$ Y-DOTA-GGNle-CycMSH $\mathrm{Hex}_{\text {hex }}$ displayed receptor-mediated binding on B16/F10 cells. Approximately $94 \%$ of ${ }^{90} \mathrm{Y}$-DOTA-GGNle-CycMSH $\mathrm{MH}_{\text {hex }}$ uptake was blocked on B16/F10 cells $(p<0.05)$ (Fig. 2). The MC1R expression on B16/F10 cells was examined through fluorescence staining using MC1R antibody. As shown in Figure 2, MC1R-binding antibody showed substantial binding on B16/F10 cells.

The cellular internalization and efflux of ${ }^{90} \mathrm{Y}$-DOTAGGNle-CycMSH $\mathrm{CH}_{\text {hex }}$ on $16 / \mathrm{F} 10$ cells are presented in Figure 3. ${ }^{90} \mathrm{Y}$-DOTA-GGNle-CycMSH $\mathrm{MH}_{\text {hex }}$ exhibited rapid cellular internalization on B16/F1 cells. Approximately $49 \%$ and $68 \%$ of ${ }^{90} \mathrm{Y}$-DOTA-GGNle-CycMSH $\mathrm{CH}_{\text {hex }}$ activity were internalized in the B16/F10 cells after $40 \mathrm{~min}$ and $2 \mathrm{~h}$ incubation, respectively. Cellular efflux of ${ }^{90} \mathrm{Y}$-DOTAGGNle-CycMSH $\mathrm{MHx}_{\text {hex }}$ demonstrated that $92 \%$ of the ${ }^{90} \mathrm{Y}$ activity remained inside the B16/F10 cells $2 \mathrm{~h}$ after incubating cells in culture medium at $25^{\circ} \mathrm{C}$.

The biodistribution result of ${ }^{90}$ Y-DOTA-GGNle$\mathrm{CycMSH}_{\text {hex }}$ on B16/F10 melanoma-bearing mice is presented in Table 1 . The B16/F10 tumor uptake was $20.73 \% \pm 7.99 \%$ and $19.93 \% \pm 5.73 \% \mathrm{ID} / \mathrm{g}$ at 0.5 and $2 \mathrm{~h}$ postinjection, respectively. ${ }^{90} \mathrm{Y}$-DOTA-GGNle-CycMSH $\mathrm{CH}_{\mathrm{hex}}$ exhibited prolonged retention in B16/F10 tumor, with $14.8 \% \pm 4.61 \%$ and $6.69 \% \pm 1.85 \%$ ID/g at 4 and $24 \mathrm{~h}$ postinjection, respectively. The coinjection of nonradioactive NDP-MSH blocked $94 \%$ of the tumor uptake at $2 \mathrm{~h}$ postinjection, demonstrating that the tumor uptake was MC1R mediated. As shown in Figure 4, MC1R-binding antibody displayed substantial binding on B16/F10 tumor sections. ${ }^{90}$ Y-DOTA-GGNle-CycMSH $\mathrm{MH}_{\text {hex }}$ displayed a rapid urinary clearance, with $\sim 91 \%$ of the injected activity being washed out of the body by $2 \mathrm{~h}$ postinjection. The accumulation of ${ }^{90} \mathrm{Y}$-DOTA-GGNle-CycMSH $\mathrm{Hex}_{\text {hex }}$ in normal organs was $<1 \% \mathrm{ID} / \mathrm{g}$ except in kidneys. The renal uptake was $12.68 \% \pm 5.2 \%, 7.44 \% \pm 1.85 \%$, and $7.75 \% \pm 1.59 \% \mathrm{ID} / \mathrm{g}$ at $0.5,2$, and $4 \mathrm{~h}$ postinjection, respectively, and decreased to $5.23 \% \pm 1.76 \% \mathrm{ID} / \mathrm{g}$ at $24 \mathrm{~h}$ postinjection. The coinjection of NDP-MSH did not significantly reduce the renal uptake $(p>0.05)$, indicating that the renal uptake of ${ }^{90} \mathrm{Y}$-DOTAGGNle-CycMSH $\mathrm{MH}_{\text {hex }}$ was not receptor mediated. ${ }^{90}$ Y-DOTAGGNle-CycMSH $_{\text {hex }}$ exhibited high tumor/blood and tumor/ normal organ uptake ratios were demonstrated as early as $0.5 \mathrm{~h}$ postinjection.

The representative maximum intensity projection bremsstrahlung SPECT image of B16/F10 melanoma-bearing mouse is presented in Figure 5. The B16/F10 flank melanoma lesions could be visualized by collecting bremsstrahlung photons from ${ }^{90} \mathrm{Y}$-DOTA-GGNle-CycMSH $\mathrm{CHex}_{\text {at }} 2 \mathrm{~h}$ postinjection. However, as shown in Figure 5, the scattered photons over the body were substantially collected by SPECT, thus decreasing the contrast of tumor to normal organ.

\section{Discussion}

The remarkable first-in-human images of patients with metastatic melanomas highlighted the clinical relevance of 
FIG. 1. Schematic structure of ${ }^{90} \mathrm{Y}$-DOTA-GGNle$\mathrm{CycMSH}_{\text {hex }}$.

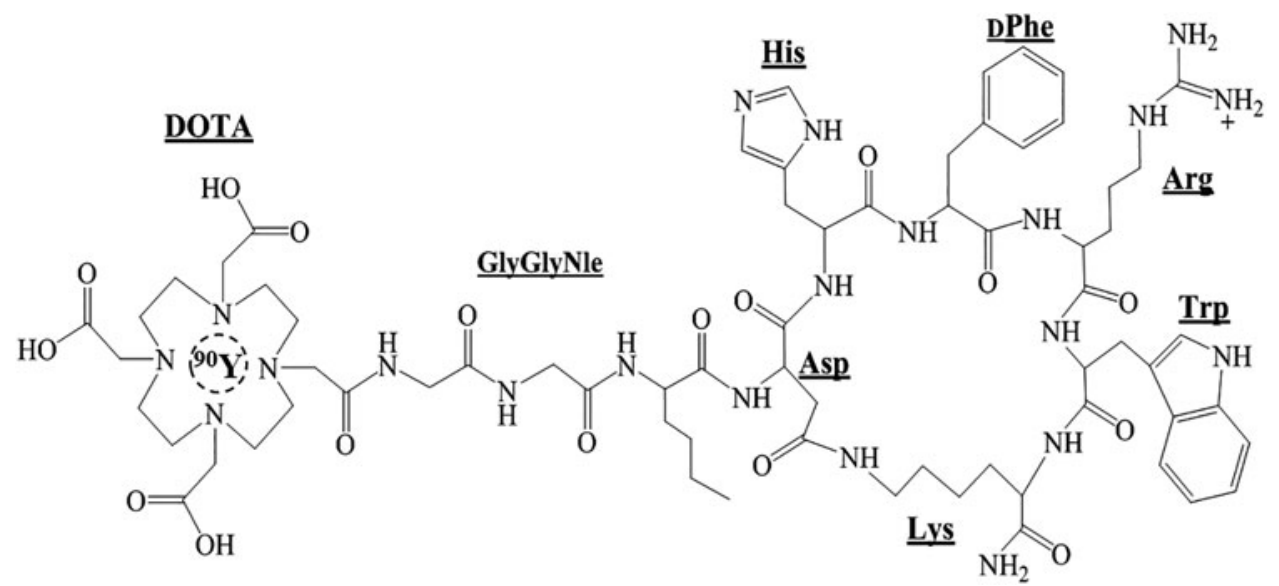

$\mathrm{MC} 1 \mathrm{R}$ as a molecular target for melanoma imaging and therapy. ${ }^{13}$ We have been interested in developing MC1Rtargeted therapeutic peptides for melanoma therapy. ${ }^{14,16}$ Both ${ }^{177} \mathrm{Lu}$ and ${ }^{90} \mathrm{Y}$ are attractive $\beta$-emitters with different half-lives and $\beta$-energy levels. ${ }^{177} \mathrm{Lu}$ has a half-life of 6.7 days with low-energy $\beta$-particles $(0.479 \mathrm{MeV})$, whereas ${ }^{90} \mathrm{Y}$ has a half-life of 2.7 days with high-energy $\beta$-particles (2.3 $\mathrm{MeV})$. Meanwhile, ${ }^{177} \mathrm{Lu}$ also emits $\gamma$-rays (113 and $208 \mathrm{keV}$ ) that can be used for imaging, while ${ }^{90} \mathrm{Y}$ is a pure $\beta$-emitter. We previously reported promising melanoma-targeting and imaging properties of ${ }^{177} \mathrm{Lu}$-DOTA-GGNle-CycMSH $\mathrm{H}_{\text {hex }} \cdot{ }^{14} \mathrm{In}$ this study, we examined the biodistribution of ${ }^{90} \mathrm{Y}$-DOTAGGNle-CycMSH $_{\text {hex }}$ on B16/F10 melanoma-bearing C57 mice. We selected B16/F10 melanoma cells for this study because they are highly metastatic and can readily form pulmonary melanoma metastases when injected into the tail veins of $\mathrm{C} 57$ mice. ${ }^{17}$ Favorable melanoma-targeting property of ${ }^{90}$ Y-DOTA-GGNle-CycMSH hex will underscore the potential of utilizing DOTA-GGNle-CycMSH ${ }_{\text {hex }}$ to deliver both ${ }^{177} \mathrm{Lu}$ and ${ }^{90} \mathrm{Y}$ to address the tumor size and burden when needed.

${ }^{90}$ Y-DOTA-GGNle-CycMSH ${ }_{\text {hex }}$ displayed MC1R-specific binding, and exhibited rapid cellular internalization and prolonged efflux on B16/F10 melanoma cells. The change of radionuclide from ${ }^{177} \mathrm{Lu}$ to ${ }^{90} \mathrm{Y}$ maintained similar melanoma-targeting property. For instance, the tumor uptake of ${ }^{90}$ Y-DOTA-GGNle-CycMSH ${ }_{\text {hex }}$ was $20.73 \% \pm 7.99 \%$ and $19.93 \% \pm 5.73 \% \mathrm{ID} / \mathrm{g}$ at 0.5 and $2 \mathrm{~h}$ postinjection, respectively, whereas the tumor uptake of ${ }^{177}$ Lu-DOTA-GGNle$\mathrm{CycMSH}_{\text {hex }}$ was $20.25 \% \pm 4.59 \%$ and $20.63 \% \pm 6.27 \% \mathrm{ID} / \mathrm{g}$ at 0.5 and $2 \mathrm{~h}$ postinjection, respectively. Meanwhile, ${ }^{90}$ Y-DOTA-GGNle-CycMSH $_{\text {hex }}$ and ${ }^{177}$ Lu-DOTA-GGNle-
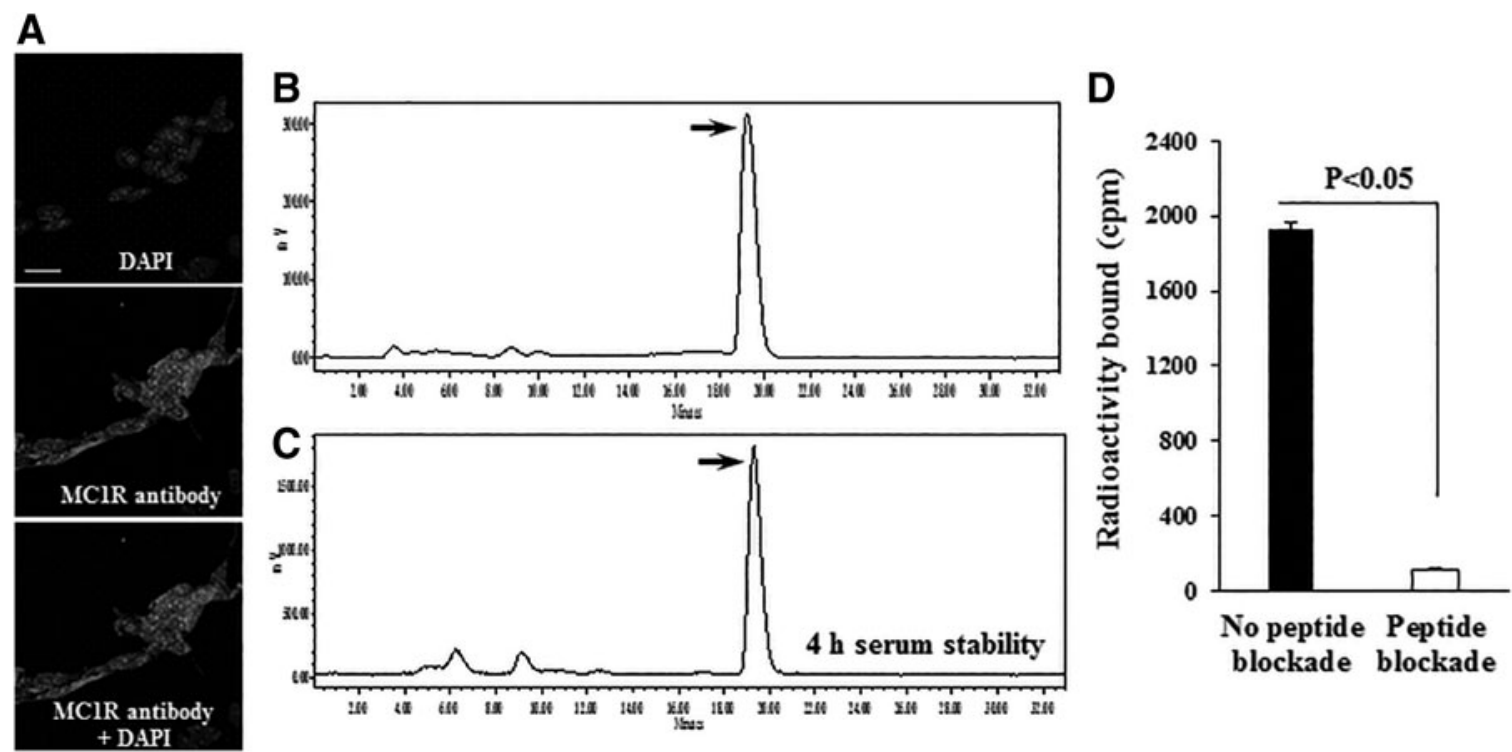

FIG. 2. Fluorescence staining of MC1Rs on B16/F10 melanoma cells (A) using MC1R antibody (white). The nuclei were stained with DAPI (grey). The microscopic images were acquired by confocal laser microscopy at $100 \times$ magnification. Scale bar, $20 \mu \mathrm{m}$. Radioactive HPLC profile of ${ }^{90} \mathrm{Y}$-DOTA-GGNle-CycMSH $\mathrm{CH}_{\text {ex }}\left(\mathbf{B}, T_{\mathrm{R}}=19.3 \mathrm{~min}\right)$ and its mouse serum stability (C) after $4 \mathrm{~h}$ incubation at $37^{\circ} \mathrm{C}$. Arrows indicate the original compound of ${ }^{90} \mathrm{Y}-\mathrm{DOTA}-\mathrm{GGNle-CycMSH} \mathrm{H}_{\text {hex }}$. Specific binding of ${ }^{90}$ Y-DOTA-GGNle-CycMSH ${ }_{\text {hex }}$ on B16/F10 (D) cells with or without peptide blockade. DAPI, 4',6diamidino-2-phenylindole; HPLC, high-performance liquid chromatography; MC1R, melanocortin-1 receptor. 
A

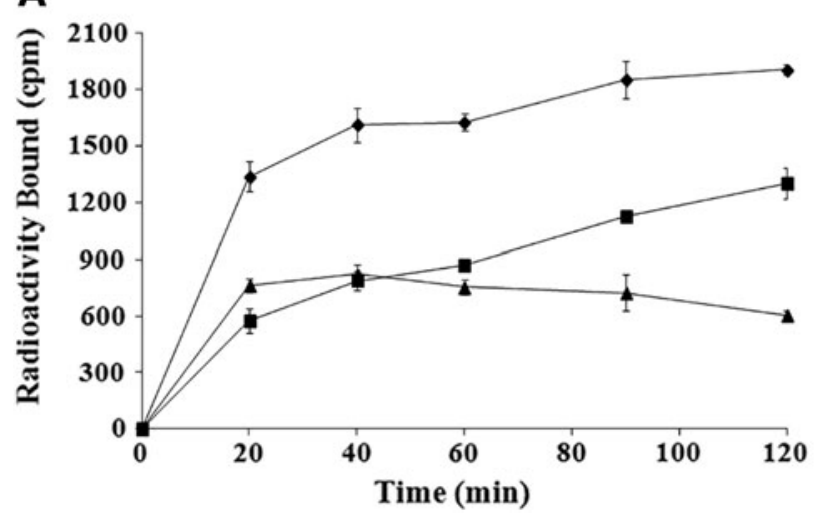

B

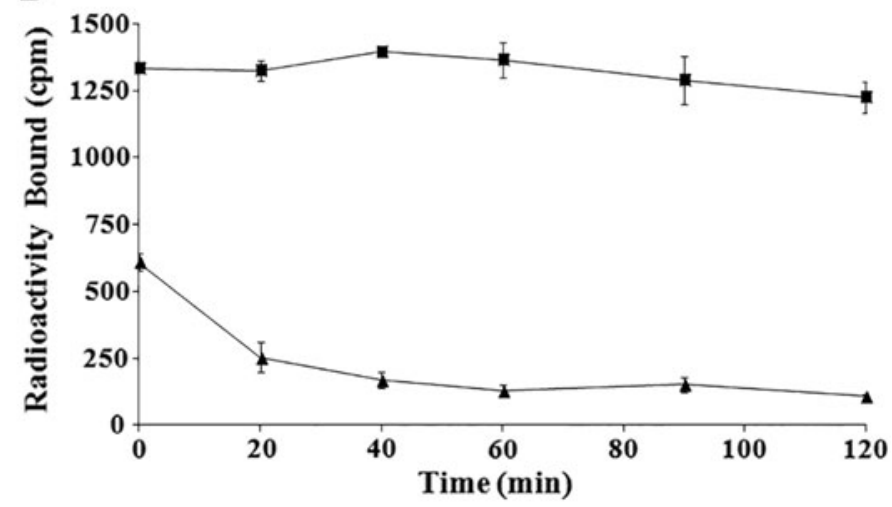

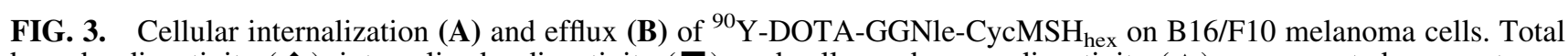
bound radioactivity $(\bullet)$, internalized radioactivity $(\boldsymbol{\square})$, and cell membrane radioactivity $(\boldsymbol{\Delta})$ are presented as counts per minute.

$\mathrm{CycMSH}_{\text {hex }}$ displayed similar fast urinary clearance. The accumulation of both peptides was low in normal organs except in kidneys that could be potential dose-limiting organs in melanoma therapy studies. Nonetheless, the rapid accumulation and prolonged retention of ${ }^{90} \mathrm{Y}$-DOTA-GGNle$\mathrm{CycMSH}_{\text {hex }}$ highlighted its potential for melanoma therapy in future studies.

Both ${ }^{90}$ Y-DOTA-Re( $\left(\operatorname{Arg}^{11}\right) \mathrm{CCMSH}$ and ${ }^{90} \mathrm{Y}$-DOTA$\mathrm{Re}\left(\mathrm{Glu}^{2}, \mathrm{Arg}^{11}\right) \mathrm{CCMSH}$ were reported to target MC1Rs for potential melanoma therapy. ${ }^{18,19}$ Their B16/F1 melanoma uptake was $25.7 \% \pm 4.64 \%$ and $11.71 \% \pm 1.32 \% \mathrm{ID} / \mathrm{g}$ at $2 \mathrm{~h}$ postinjection, respectively. Although the tumor uptake of ${ }^{90}$ Y-DOTA-
$\operatorname{Re}\left(\mathrm{Glu}^{2}, \mathrm{Arg}^{11}\right) \mathrm{CCMSH}$ was lower than that of ${ }^{90} \mathrm{Y}$-DOTA$\operatorname{Re}\left(\mathrm{Arg}^{11}\right) \mathrm{CCMSH},{ }^{90} \mathrm{Y}-\mathrm{DOTA}-\operatorname{Re}\left(\mathrm{Glu}^{2}, \mathrm{Arg}^{11}\right) \mathrm{CCMSH}$ displayed higher tumor to kidney uptake ratio than ${ }^{90} \mathrm{Y}$-DOTA-Re $\left(\mathrm{Arg}^{11}\right) \mathrm{CCMSH}$. Interestingly, ${ }^{90} \mathrm{Y}$-DOTAGGNle-CycMSH $\mathrm{Mex}_{\text {hex }}$ exhibited the highest tumor to kidney uptake ratio (2.68 at $2 \mathrm{~h}$ postinjection) among these peptides because of its high tumor uptake and low renal uptake. The improved tumor to kidney uptake ratio of ${ }^{90} \mathrm{Y}$-DOTA-

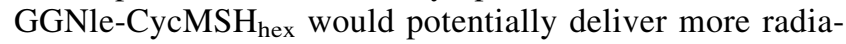
tion to tumor without increasing the radiation to kidneys.

The melanoma lesions could be clearly visualized by SPECT using ${ }^{177}$ Lu-DOTA-GGNle-CycMSH ${ }_{\text {hex }}$ as an imaging

Table 1. Biodistribution of ${ }^{90}$ Y-DOTA-GGNle-CyCMSH ${ }_{\text {Hex }}$ on B16/F10 Murine Melanoma-Bearing C57 Mice

\begin{tabular}{|c|c|c|c|c|c|}
\hline Tissues & $0.5 h$ & $2 h$ & $2 \mathrm{~h}$ blockade & $4 h$ & $24 h$ \\
\hline \multicolumn{6}{|c|}{ Percent injected dose/gram (\% ID/g) } \\
\hline Tumor & $20.73 \pm 7.99$ & $19.93 \pm 5.73$ & $1.20 \pm 1.04^{\mathrm{a}}$ & $14.8 \pm 4.61$ & $6.69 \pm 1.85$ \\
\hline Brain & $0.10 \pm 0.02$ & $0.05 \pm 0.03$ & $0.03 \pm 0.03$ & $0.03 \pm 0.02$ & $0.03 \pm 0.02$ \\
\hline Blood & $2.76 \pm 0.69$ & $0.36 \pm 0.15$ & $0.25 \pm 0.12$ & $0.22 \pm 0.12$ & $0.06 \pm 0.07$ \\
\hline Heart & $1.28 \pm 0.51$ & $0.14 \pm 0.10$ & $0.13 \pm 0.05$ & $0.16 \pm 0.09$ & $0.11 \pm 0.07$ \\
\hline Lung & $3.06 \pm 1.05$ & $0.42 \pm 0.08$ & $0.19 \pm 0.12$ & $0.27 \pm 0.05$ & $0.13 \pm 0.15$ \\
\hline Liver & $0.99 \pm 0.19$ & $0.63 \pm 0.14$ & $0.39 \pm 0.12$ & $1.20 \pm 1.34$ & $0.60 \pm 0.20$ \\
\hline Skin & $2.48 \pm 1.05$ & $0.78 \pm 0.29$ & $0.10 \pm 0.12$ & $0.53 \pm 0.29$ & $0.37 \pm 0.18$ \\
\hline Spleen & $1.4 \pm 1.36$ & $0.24 \pm 0.17$ & $0.15 \pm 0.12$ & $0.31 \pm 0.17$ & $0.53 \pm 0.42$ \\
\hline Stomach & $1.36 \pm 0.4$ & $0.87 \pm 0.47$ & $0.15 \pm 0.06$ & $0.61 \pm 0.27$ & $0.76 \pm 0.52$ \\
\hline Kidneys & $12.68 \pm 5.2$ & $7.44 \pm 1.85$ & $4.99 \pm 0.69$ & $7.75 \pm 1.59$ & $5.23 \pm 1.76$ \\
\hline Muscle & $0.73 \pm 0.51$ & $0.19 \pm 0.13$ & $0.10 \pm 0.10$ & $0.22 \pm 0.31$ & $0.15 \pm 0.14$ \\
\hline Pancreas & $0.61 \pm 0.43$ & $0.18 \pm 0.05$ & $0.06 \pm 0.07$ & $0.14 \pm 0.09$ & $0.16 \pm 0.14$ \\
\hline Bone & $1.37 \pm 0.44$ & $0.41 \pm 0.18$ & $0.15 \pm 0.14$ & $0.44 \pm 0.34$ & $0.2 \pm 0.04$ \\
\hline \multicolumn{6}{|c|}{ Percent injected dose (\% ID) } \\
\hline Intestines & $1.42 \pm 0.38$ & $0.57 \pm 0.22$ & $0.33 \pm 0.15$ & $1.36 \pm 1.16$ & $1.92 \pm 3.51$ \\
\hline Urine & $75.42 \pm 6.72$ & $90.62 \pm 1.79$ & $96.77 \pm 0.37$ & $89.45 \pm 4.25$ & $93.01 \pm 4.43$ \\
\hline \multicolumn{6}{|c|}{ Uptake ratio of tumor/normal tissue } \\
\hline Tumor/blood & 7.51 & 55.36 & 4.8 & 67.27 & 111.5 \\
\hline Tumor/kidney & 1.63 & 2.68 & 0.24 & 1.91 & 1.28 \\
\hline Tumor/lung & 6.77 & 47.45 & 6.32 & 54.81 & 51.46 \\
\hline Tumor/liver & 20.94 & 31.63 & 3.08 & 12.33 & 11.15 \\
\hline Tumor/muscle & 28.4 & 104.89 & 12.0 & 67.27 & 44.6 \\
\hline
\end{tabular}

The data were presented as percentage injected dose/gram or as percentage injected dose $(m e a n \pm S D, N=5)$.

${ }^{a} p<0.05$ for determining significance of differences in tumor and kidney uptake between ${ }^{90} \mathrm{Y}-\mathrm{DOTA}-\mathrm{GGNle}-\mathrm{CyCMSH}_{\mathrm{hex}}$ with or without peptide blockade at $2 \mathrm{~h}$ postinjection. 


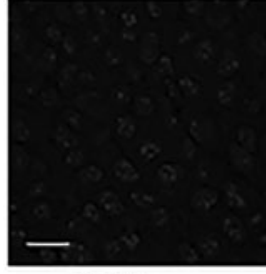

DAPI

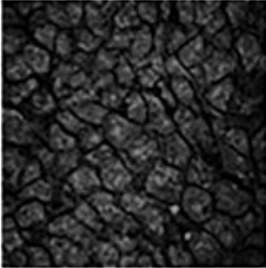

MClR antibody MClR antibody + DAPI
FIG. 4. Fluorescence staining of MC1Rs on B16/F10 tumor section using MC1R antibody (white). The nuclei were stained with DAPI (grey). The microscopic images were acquired by confocal laser microscopy at $100 \times$ magnification. Scale bar, $20 \mu \mathrm{m}$. DAPI, 4',6-diamidino-2-phenylindole; $\mathrm{MC} 1 \mathrm{R}$, melanocortin-1 receptor.

agent due to the $\gamma$-rays from ${ }^{177} \mathrm{Lu} .{ }^{14}$ Although ${ }^{90} \mathrm{Y}$ is a pure $\beta$-emitter, Bremsstrahlung photons could be produced by interaction of the $\beta$-particles of ${ }^{90} \mathrm{Y}$ with tissue. Thus, both Bremsstrahlung SPECT and PET of ${ }^{90} \mathrm{Y}$ have been investigated and reported in the literature. ${ }^{20-23}$ In this study, we

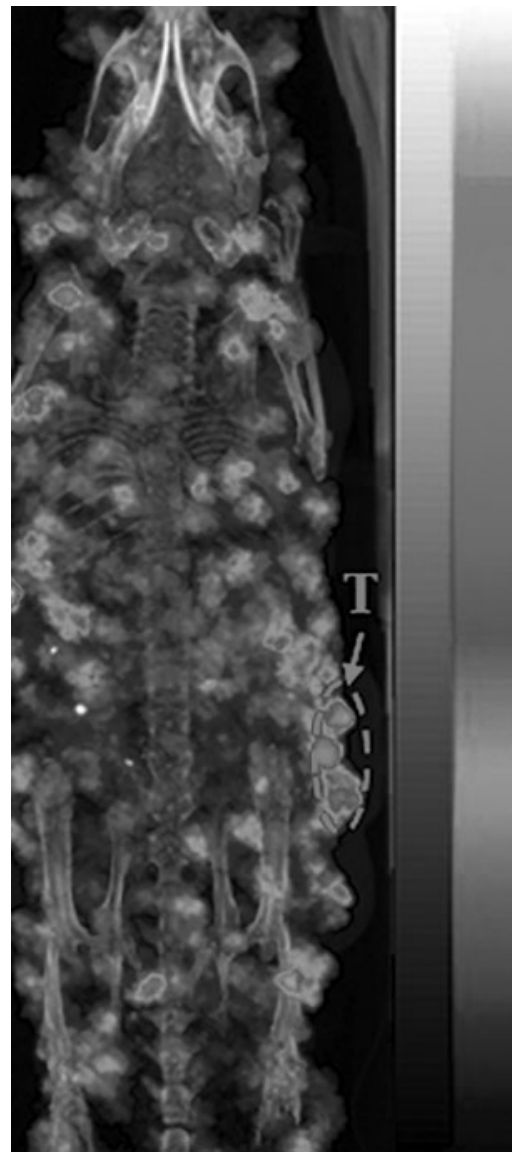

FIG. 5. Representative maximum intensity projection Bremsstralung SPECT/CT image of a B16/F10 flank melanoma-bearing C57 mouse using ${ }^{90}$ Y-DOTA-GGNle$\mathrm{CycMSH}_{\text {hex }}$ as an imaging probe at $2 \mathrm{~h}$ postinjection. Melanoma lesions are highlighted with an arrow on the image. white and black indicate high and low activity on the scale bar, respectively. SPECT, single photon emission computed tomography. examined the Bremsstrahlung SPECT of ${ }^{90}$ Y-DOTA-GGNle$\mathrm{CycMSH}_{\text {hex }}$ on melanoma-bearing mice using a small energy window of $126.5-155.7 \mathrm{keV}$. Although the flank melanoma lesions could be visualized by collecting bremsstrahlung photons from ${ }^{90} \mathrm{Y}$-DOTA-GGNle-CycMSH $\mathrm{MHx}_{\text {hex }}$, the presence of scattered photons in the image resulted in degradation in contrast to tumor to normal organ. The challenge in imaging

${ }^{90} \mathrm{Y}$ using SPECT is due to its continuous Bremsstrahlung spectrum with energies up to $2.3 \mathrm{MeV}$ and the absence of a photopeak, making the traditional small energy windowbased scatter rejection ineffective.

Yttrium-86 is an attractive PET radionuclide that can be produced by a cyclotron through the ${ }^{86} \mathrm{Sr}(\mathrm{p}, \mathrm{n}){ }^{86} \mathrm{Y}$ reaction. It has a half-life of $14.7 \mathrm{~h}$ and can serve as an imaging surrogate for ${ }^{90} \mathrm{Y}$ to form a true matched-pair theranostic radionuclide. ${ }^{86}$ Y-DOTA-ReCCMSH(Arg ${ }^{11}$ ) has been developed to target MC1Rs for melanoma imaging. It exhibited rapid high B16/F1 melanoma uptake $(11.87 \% \pm 3.31 \% \mathrm{ID} / \mathrm{g}$ at $0.5 \mathrm{~h}$ postinjection) and low accumulation in normal organs expect in kidneys. ${ }^{24}$ The PET images of melanoma-bearing mice using ${ }^{86}$ Y-DOTA-ReCCMSH( $\mathrm{Arg}^{11}$ ) clearly demonstrated its melanoma imaging potential due to its high tumor concentration and low nontarget tissue accumulation. However, the availability of ${ }^{86} \mathrm{Y}$ can potentially be a limiting factor since it is not a commercial radionuclide. Alternatively, ${ }^{68} \mathrm{Ga}$-DOTAGGNle-CycMSH $\mathrm{Hex}_{\text {he }}$ can be used to monitor the response when examining the therapeutic efficacy of ${ }^{90} \mathrm{Y}$-DOTA-GGNle$\mathrm{CycMSH}_{\text {hex }}$ in the future.

\section{Conclusions}

${ }^{90}$ Y-DOTA-GGNle-CycMSH $_{\text {hex }}$ displayed MC1R targeting and specificity on B16/F10 melanoma cells and tumors. The favorable melanoma-targeting property and fast urinary clearance of ${ }^{90} \mathrm{Y}$-DOTA-GGNle-CycMSH $\mathrm{H}_{\text {hex }}$ warranted its evaluation for melanoma therapy in future studies.

\section{Acknowledgment}

We thank Dr. Fabio Gallazzi for his technical assistance.

\section{Disclosure Statement}

No competing financial interests exist.

\section{Funding Information}

This work was supported in part by the NIH grant R01CA225837 and University of Colorado Denver startup fund. Microscopy imaging experiments were performed in the University of Colorado Anschutz Medical Campus Advance Light Microscopy Core supported in part by NIH/NCATS Colorado CTSI Grant Number UL1 TR001082.

\section{References}

1. Siegel RL, Miller KD, Jemal A. Cancer Statistics, 2019. CA Cancer J Clin 2019;69:7.

2. Chapman PB, Hauschild A, Robert C, et al.; BRIM-3 Study Group. Improved survival with vemurafenib in melanoma with BRAF V600E mutation. N Engl J Med 2011;364:2507.

3. Sosman JA, Kim KB, Schuchter L, et al. Survival in BRAF V600-mutant advanced melanoma treated with vemurafenib. N Engl J Med 2012;366:707. 
4. Hodi FS, O'Day SJ, McDermott DF, et al. Improved survival with ipilimumab in patients with metastatic melanoma. N Engl J Med 2010;363:711.

5. Weber JS, O'Day S, Urba W, et al. Phase I/II study of ipilimumab for patients with metastatic melanoma. J Clin Oncol 2008;26:5950.

6. Topalian SL, Sznol M, McDermott DF, et al. Survival, durable tumor remission, and long-term safety in patients with advanced melanoma receiving nivolumab. J Clin Oncol 2014;32:1020.

7. Tatro JB, Wen Z, Entwistle ML, et al. Interaction on an $\alpha$ melanocyte stimulating hormone-diptheria toxin fusion protein with melanotropin receptors in human metastases. Cancer Res 1992;52:2545.

8. Siegrist W, Solca F, Stutz S, et al. Characterization of receptors for alpha-melanocyte-stimulating hormone on human melanoma cells. Cancer Res 1989;49:6352.

9. Tatro JB, Reichlin S. Specific receptors for alphamelanocyte-stimulating hormone are widely distributed in tissues of rodents. Endocrinology 1987;121:1900.

10. Chen J, Cheng Z, Hoffman TJ, et al. Melanoma-targeting properties of ${ }^{99 \mathrm{~m}}$ Technetium-labeled cyclic $\alpha$-melanocytestimulating hormone peptide analogues. Cancer Res 2000;60: 5649.

11. Miao Y, Whitener D, Feng W, et al. Evaluation of the human melanoma targeting properties of radiolabeled alphamelanocyte stimulating hormone peptide analogues. Bioconjug Chem 2003;14:1177.

12. Guo H, Shenoy N, Gershman BM, et al. Metastatic melanoma imaging with an ${ }^{111}$ In-labeled lactam bridge-cyclized alpha-melanocyte stimulating hormone peptide. Nucl Med Biol 2009;36:267.

13. Yang J, Xu J, Gonzalez R, et al. ${ }^{68}$ Ga-DOTA-GGNle$\mathrm{CycMSH}_{\text {hex }}$ targets the melanocortin-1 receptor for melanoma imaging. Sci Transl Med 2018;10:eaau4445.

14. Guo H, Miao Y. Melanoma targeting property of a Lu-177labeled lactam bridge-cyclized alpha-MSH peptide. Bioorg Med Chem Lett 2013;23:2319.
15. Guo H, Yang J, Gallazzi F, et al. Effects of the amino acid linkers on melanoma-targeting and pharmacokinetic properties of indium-111-labeled lactam bridge-cyclized $\alpha$ MSH peptides. J Nucl Med 2011;52:608.

16. Miao Y, Shelton T, Quinn TP. Therapeutic efficacy of a ${ }^{177} \mathrm{Lu}$ labeled DOTA conjugated $\alpha$-melanocyte stimulating hormone peptide in a murine melanoma-bearing mouse model. Cancer Biother Radiopharm 2007;22:333.

17. Yang J, Xu J, Cheuy L, et al. Novel Pb-203-labeled lactamcyclized alpha-melanocyte-stimulating hormone peptide for melanoma imaging. Mol Pharm 2019;16:1694.

18. Miao Y, Hoffman TJ, Quinn TP. Tumor targeting properties of ${ }^{90} \mathrm{Y}$ and ${ }^{177} \mathrm{Lu}$ labeled alpha-melanocyte stimulating hormone peptide analogues in a murine melanoma model. Nucl Med Biol 2005;32:485.

19. Miao Y, Fisher DR, Quinn TP. Reducing renal uptake of ${ }^{90} \mathrm{Y}$ and ${ }^{177} \mathrm{Lu}$ labeled alpha-melanocyte stimulating hormone peptide analogues. Nucl Med Biol 2006;33: 723.

20. Elschot M, Vermolen BJ, Lam M, et al. Quantitative comparison of PET and Bremsstrahlung SPECT for imaging the in vivo yttrium-90 microsphere distribution after liver radioembolization. PLoS One 2013;8:e55742.

21. Porter CA, Bradley KM, Hippeläinen ET, et al. Phantom and clinical evaluation of the effect of full Monte Carlo collimator modelling in post-SIRT yttrium-90 Bremsstrahlung SPECT imaging. EJNMMI Res 2018;8:7.

22. Yue J, Mauxion T, Reyes DK, et al. Comparison of quantitative Y-90 SPECT and non-time-of-flight PET imaging in post-therapy radioembolization of liver cancer. Med Phys 2016;43:5779.

23. Barber TW, Cherk MH, Powell A, et al. Correlation of clinical outcomes with bremsstrahlung and Y-90 PET/CT imaging findings following Y-90 radiosynoviorthesis: A prospective study. EJNMMI Res 2016;6:45.

24. McQuade P, Miao Y, Yoo J, et al. Imaging of melanoma using ${ }^{64} \mathrm{Cu}$ and ${ }^{86} \mathrm{Y}$-DOTA-ReCCMSH(Arg $\left.{ }^{11}\right)$, a cyclized peptide analogue of (-MSH. J Med Chem 2005;48:2985. 